\title{
The Challenge of Glaucoma Surgery in the Cataract Patient - Where Are We Now?
}

\author{
Mildred MG Olivier \\ Professor, Department of Surgery, Division of Ophthalmology, Chicago Medical School, Chicago, uS
}

DOI: http://doi.org/10.17925/EOR.2015.09.02.128

\begin{abstract}
Choosing the best approach for cataract removal in glaucoma patients depends on the extent of glaucoma-related damage and the medications in use to control IOP. Micro-invasive glaucoma surgery offers additional options for patients needing combination surgery without altering the conjunctiva.
\end{abstract}

\section{Keywords}

Glaucoma surgery, cataract patients, phacoemulsification, trabeculectomy, micro-invasive glaucoma surgery

Disclosure: Mildred MG Olivier has nothing to disclose in relation to this article. No funding was received in the publication of this article.

open Access: This article is published under the Creative Commons Attribution Noncommercial License, which permits any non-commercial use, distribution, adaptation and reproduction provided the original author(s) and source are given appropriate credit.

Received: 1 December 2015 Published Online: 23 December 2015 Citation: European Ophthalmic Review, 2015;9(2):128-9

Correspondence: Mildred MG Olivier, Midwest Glaucoma Center, P.C., 1555 N Barrington Road, DOB \#1, Suite 110, Hoffman Estates, Illinois 60169-1062, US.

E: receptionist@midwestglaucoma.com

Of the approximately 3 million cataract surgeries performed in the US each year, epidemiologists predict that $10 \%$ will have ocular hypertension or open angle glaucoma (OAG). Ophthalmologists must be ready to handle those surgeries as well as the 100,000 stand-alone glaucoma procedures.

Glaucoma patients present special challenges in cataract surgery. How you proceed with cataract removal depends on the extent of glaucomarelated damage to the optic disc and the medications in use to control intraocular pressure (IOP). Patients with impaired outflow facility are more likely to have post-operative IOP spikes; therefore, combining cataract and glaucoma surgery is often recommended.

With myriad surgical options available for cataract removal, how do you choose what's best for your glaucoma patient? The options include cataract surgery alone, filtration surgery (trabeculectomy [trab] or glaucoma drainage device [GDD]) first followed by phacoemulsification (phaco), or combining the two in one operation.

Choosing the best option depends on a number of factors. How severe is the patient's glaucoma, and how well will the optic nerve tolerate IOP spikes associated with surgery? How many medications does the patient take before surgery? What is the person's age and ability to follow a medical regimen? Does the patient have a desire to reduce their dependence on medical therapy? The patient's medical history, ocular or systemic co-morbidities and glaucoma phenotype should be considered.

Patients and doctors see the appeal of combined procedures to cut down on the number of trips to the operating room. There are trade-offs, however. When considering whether to perform cataract surgery alone or to combine it with filtering surgery, studies show that combining procedures increases surgical risk and recovery time.
Cataract surgery alone may reduce IOP in both glaucomatous and non-glaucomatous eyes. The mechanisms of reduced IOP may be due to a wider anterior chamber angle, or the pull of the iris on the ciliary body/scleral spur with widening of the trabecular beams. It could also result from decreased aqueous production from low-grade post-operative inflammation. In OAG, the IOP-lowering effect may be transient. However, patients with angle closure glaucoma (ACG), or narrow angles, may achieve a substantial and sustained reduction of IOP due to relief of pupillary block.

With combined phaco-trab, studies have shown decreased risk of postoperative IOP spikes, lower average IOP of $8 \mathrm{mmHg}$ after 1 to 2 years and a $1.5 \%$ reduction in medications compared with patients' baseline measurements. In terms of the surgical approach, two-site, (clear cornea phaco and superior trab), may be better than same-site (scleral tunnel converted to a trab flap). Using anti-metabolite has been shown to improve the outcome of same site surgery.

In patients with uncontrolled glaucoma, it might be advisable to perform a trab well in advance of cataract surgery. Trab alone controls IOP better than a phaco-trab. Studies found a reduction of 10.3 to $15.8 \mathrm{mmHg}$ after trab alone compared with 6.8 to $9.2 \mathrm{mmHg}$ after phaco-trab. However, trab is itself cataractogenic, with the accompanying intraocular inflammation, flat $\mathrm{AC}$, as well as the steroids required post-operatively. The Advanced Glaucoma Intervention Study found a $78 \%$ risk of visually significant cataract within 5 years of trab. A flat anterior chamber and marked inflammation increased the risk.

Cataract patients with pre-existing filtering blebs present another concern as they may not work as well after cataract surgery. Among patients with blebs, one study found $30.4 \%$ required additional medication or bleb needling and $9.6 \%$ needed additional glaucoma 
surgery. Other studies reported no IOP difference among patients receiving clear corneal phaco. Post-phaco bleb function may also be maintained with the help of intraoperative subconjunctival 5 -fluorouracil (5-FU) or mitomycin injection.

Another possibility for patients with advanced, uncontrolled glaucoma is trab with express shunt. The shunt provides a uniform ostomy connection with the anterior chamber. Since iridectomy is unnecessary there is less inflammation.

Micro-invasive glaucoma surgery (MIGS) offers additional options for patients requiring combination surgery. MIGS procedures are performed through a clear corneal incision without altering the conjunctiva. Candidates for these procedures typically have mild to moderate OAG with target pressures in the mid-teens with or without long-term antiglaucoma medications (e.g. endoscopic cyclophotocoagulation, Trabectome and istent trabecular micro-bypass shunt).
The discussion of cataract surgery in glaucoma patients would be incomplete without considering post-operative IOP spikes. Retained lens material or viscoelastic, steroid response or secondary pigment dispersion syndrome are some causes.

In summary, patients whose glaucoma is controlled with one or two medications may maintain good IOP after phaco alone. Consider combined phaco-trab or glaucoma drainage devices for patients needing three or more medications, or who are at high risk of post-op IOP spikes. 'Bleb free' glaucoma surgery (MIGS) can be safely combined with phaco. Surgeons must, however, be meticulous with viscoelastic removal to prevent IOP spikes, and should be wary of surgical challenges such as small pupils or weak zonules.

There are now more surgical options for glaucoma patients undergoing cataract surgery. With the advances in phaco and the expansion of angle surgeries, one day we hope to have the perfect formula for success. 